\title{
Thickness Uniformity Dependence on Polymer Viscosity in Silver-Nanowire-Embedded Flexible and Transparent Electrodes
}

\author{
Moonsoo Chae ${ }^{1}$, Dongwook Ko ${ }^{1}$, Yoohan Ma ${ }^{1}$, Sungjin Jo ${ }^{2}$, Dong Choon Hyun ${ }^{3}$, \\ Hyeon-Ju Oh ${ }^{4, *}$ and Jongbok Kim ${ }^{1,4, *}$
}

1 Department of Materials Science and Engineering, Kumoh National Institute of Technology, Gumi, Gyeongbuk 39177, Korea; cha68533@naver.com (M.C.); duko1293@gmail.com (D.K.); john931023@gmail.com (Y.M.)

2 School of Architectural, Civil, Environmental, and Energy Engineering, Kyungpook National University, Daegu 41566, Korea; sungjin@knu.ac.kr

3 Department of Polymer Science and Engineering, Kyungpook National University, Daegu 41566, Korea; dong.hyun@knu.ac.kr

4 Advanced Materials Research Center, Kumoh National Institute of Technology, Gumi, Gyeongbuk 39177, Korea

* Correspondence: smju26@kumoh.ac.kr (H.-J.O.); jbkim@kumoh.ac.kr (J.K.); Tel.: +82-54-478-7748 (H.-J.O. \& J.K.)

Received: 14 February 2020; Accepted: 23 March 2020; Published: 25 March 2020

\begin{abstract}
We herein report the effect of the viscosity of a prepolymer solution on the thickness uniformity of silver-nanowire-embedded flexible transparent electrodes. We adopted a model system with all the prepolymer solutions possessing identical physical properties except for the viscosity and then explored the most common prepolymer solutions for silver-nanowire-embedded flexible electrodes. In all experiments, single-step spin coating was conducted to coat the prepolymer solution on silver nanowires. We found that the electrodes were thinner for lower viscosity. However, the thickness ratio between the center and edge was comparable (50-60\%) and independent of the prepolymer solution viscosity. This indicates that the viscosity does not determine the thickness uniformity, and that the coating method itself is vital to obtain films with uniform thickness. The flexible electrodes were introduced into organic solar cells. Their device performance was comparable regardless of the position of the electrodes and their thickness. This is because the thickness difference of the flexible electrodes did not affect their transmittance significantly. Thus, we conclude that although different coating approaches are needed to obtain flexible electrodes with high uniformity, the performance of optoelectronic devices on silver-nanowire-embedded flexible electrodes is independent of them.
\end{abstract}

Keywords: silver nanowire; polymer viscosity; embedding; flexible electrode; thickness uniformity

\section{Introduction}

Owing to the advances in electronics, environmental detection, obtaining extended information effortlessly, and solving complex calculations simply are now easy tasks. In their early stage, electronics materials and devices were rigid, limiting their installation and usage; the current need for more convenient devices to be used in a wide variety of applications has led to the development of flexible electronic devices [1-3]. To fabricate flexible electronic devices, all functional materials should be flexible. In particular, the properties of transparent flexible electrodes are very important in optoelectronics because these support all functional layers as a substrate [4,5]. Indium tin oxide (ITO) 
deposited on a flexible film was a popular material for flexible transparent electrodes because of its high conductivity and stability as an electrode and because of being common in rigid electronics [6-10]. However, it cracks easily when stress is applied and is not suitable for flexible electronic devices that have to be bent repeatedly [11-13]. To realize flexible devices, many candidates have been developed, including graphene, carbon nanotubes, metal nanowires, and organic conductors [14-19]. Although graphene, carbon nanotubes, and organic conductors are limited by their low area, yield, or electronic performance, metal-nanowire-based flexible transparent electrodes, especially those based on silver nanowire, have displayed high conductivity and high transmittance and facilitated easy manufacturing in a large area at a low cost [20-22].

Although the excellent performance of silver-nanowire-based transparent electrodes showed their potential as flexible electrodes, their rough surface hindered their application to electronic devices. In particular, their rough surface and sharpness cause them to break through the functional layers and contact the counter electrode, resulting in device shorting [23-27]. To prevent roughness issues, researchers have embedded conductive silver nanowires into flexible polymers [26,27]. Because the nanowires form a network inside the polymer, the surface of the electrode is flat, avoiding device shorting. In our previous research, we demonstrated silver nanowires on a flexible film with a root mean squared (RMS) roughness of $8 \mathrm{~nm}$ and silver-nanowire-embedded electrodes with an RMS roughness of $1 \mathrm{~nm}$, which improved the reliability of the construction and operation of electronic devices [28]. To embed sharp silver nanowires into a polymer, it is essential to coat a prepolymer with high viscosity on the nanowires and detach it from the substrate after curing. However, the high viscosity can induce a nonuniform thickness of the flexible electrode that can affect the electrode properties and the performance of the final device.

In this study, we controlled the viscosity of the prepolymer for embedding nanowires and investigated its effect on the thickness difference in silver-nanowire-embedded transparent flexible electrodes. We initially adopted prepolymer SU8 as a model system to construct silver-nanowire-embedded flexible electrodes because adding a solvent into SU8 changes its viscosity without affecting other properties such as its elastic modulus and strength. Although thinner films showed lower thickness difference between the edge and center, the corresponding thickness ratio was approximately $60 \%$ in all films. Moreover, when using Norland optical adhesives (NOAs, Norland Products), which are common polymers for embedding silver nanowires, the result was similar, with thinner films having a lower thickness difference. However, they showed a comparable thickness ratio of approximately $50 \%$. Although there was a slight thickness difference in the silver-nanowire-embedded electrodes, their optoelectrical device performance when constructing organic solar cells on them was comparable regardless of their location. This indicates that it is difficult to obtain flexible electrodes with uniform thickness, and that the thickness ratio is comparable regardless of the viscosity of the prepolymer when the flexible electrode is constructed by single-step spin coating. Additionally, we showed that the thickness difference is not critical for the performance of the optoelectronic devices.

\section{Materials and Methods}

\subsection{Preparation of Prepolymer Solutions with Different Viscosities}

NOAs are popular materials used to embed silver nanowires for constructing flexible transparent electrodes. However, different NOA series have different physical properties and viscosity. Therefore, we initially used SU8 as a model system to embed silver nanowires and construct nanowire-based transparent electrodes, although it is not suitable for transparent electrodes because of its yellowish color. To control the viscosity of SU8, we tuned its solid content by adding a thinner (MicroChem) to SU8 2015 (MicroChem). Because a thinner is just a solvent, it changes only the viscosity of the solution without affecting the physical properties of the polymer film obtained after curing. Specifically, for contents of thinner solvent in SU8 2015 of $0 \mathrm{~mL}, 9.4 \mathrm{~mL}, 20.86 \mathrm{~mL}$, and $41 \mathrm{~mL}$, the SU8 had viscosities of 1500 cps, 451.06 cps, 164.5 cps, and 52.38 cps, respectively. After studying the effect of viscosity in 
the model system, we tested NOA series $63,65,71$, and 73, which have viscosities ranging from 140 cps to $2500 \mathrm{cps}$ and different elastic modulus, strength, and hardness values.

\subsection{Fabrication of Silver-Nanowire-Embedded Flexible Transparent Electrodes}

Figure 1 depicts the process used to fabricate silver-nanowire-embedded flexible transparent conductive electrodes and shows a representative scanning electron microscope (SEM) image of them. We first prepared a $0.3 \mathrm{wt} \%$ silver nanowire solution dispersed in deionized water (Nanopyxis, South Korea). The nanowires in the solution had a diameter of $35 \pm 5 \mathrm{~nm}$ and length of $25 \pm 5 \mu \mathrm{m}$, generally capped by polyvinylpyrrolidone (PVP) to make a solution [29-31]. Glass substrates sized $2.5 \times 3 \mathrm{~cm}$ were cleaned by sonication in acetone and isopropyl alcohol at $60^{\circ} \mathrm{C}$ for $15 \mathrm{~min}$, followed by drying in an oven at $100{ }^{\circ} \mathrm{C}$. We then spin-coated polymethylmethacrylate (PMMA) at $3000 \mathrm{rpm}$ for $60 \mathrm{~s}$ on the glass substrates to form a releasing layer with low adhesive force. The releasing layer should have adhesive force with silver nanowires weaker than the adhesive force between the UV-curable polymer and silver nanowires for complete detachment of the silver nanowires from the releasing layer. In detail, while the glass substrate has an adhesive force of $169.5 \mathrm{~mJ} \mathrm{~m}^{-2}$ with the silver-nanowire film having a polar surface energy of $131.3 \mathrm{~mJ} \mathrm{~m}^{-2}$ and a nonpolar surface energy of $10.3 \mathrm{~mJ} \mathrm{~m}^{-2}$, the PMMA surface has an adhesive force of $112.5 \mathrm{~mJ} \mathrm{~m}^{-2}$ with identical silver-nanowire film [28]. After drying the PMMA-coated glass substrate at $180^{\circ} \mathrm{C}$ for $30 \mathrm{~s}$, the silver nanowire solution was spin-coated on it at $600 \mathrm{rpm}$ for $60 \mathrm{~s}$. After drying at $100^{\circ} \mathrm{C}$ for $1 \mathrm{~min}$, a silver nanowire network for electron conduction formed on the substrates. To embed the silver nanowires into a polymer and fabricate silver-nanowire-embedded electrodes, ultraviolet (UV)-curable SU8 or NOA polymers with different viscosities were spin-coated at 250-1000 rpm for $60 \mathrm{~s}$ and cured under UV light irradiation for $30 \mathrm{~min}$. Silver nanowires are embedded into UV-curable polymers by a principle of mechanical interlocking during the curing process $[26,28]$. The delamination of the UV-curable polymer completed the fabrication of silver-nanowire-embedded flexible and transparent electrodes. Due to the low adhesive force of the PMMA-coated surface with silver nanowire, the silver nanowires could be fully detached from the substrate $[26,28]$. In addition, light scattering was insignificant in the silver-nanowire-embedded transparent electrodes in SU8 or NOA polymers because the electrodes were very flat $[26,28]$. Then, to investigate whether the electrodes were well constructed, their surfaces were observed by SEM (JSM-6500F, Jeol).

(a)
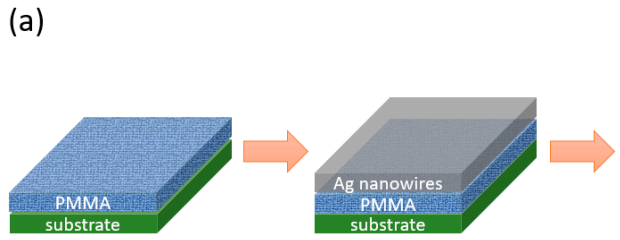

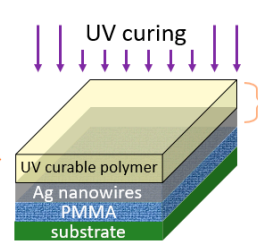

(b)

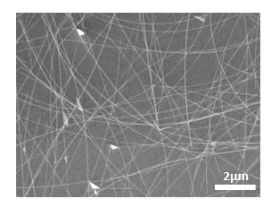

Figure 1. (a) Schematic illustration of the fabrication of silver-nanowire-embedded flexible transparent electrodes, displaying the thickness measurement locations. (b) A representative SEM image of silver-nanowire-embedded transparent electrodes.

\subsection{Characterization of Silver-Nanowire-Embedded Flexible Transparent Electrodes}

To evaluate the thickness uniformity of the silver-nanowire-embedded flexible transparent electrodes as a function of the polymer viscosity, we measured the thicknesses of flexible electrodes that were prepared with prepolymer solutions of different viscosity, coated at different spinning speeds. The thickness was measured at the center and the edge of the electrodes using a digital Vernier caliper (Slyzone, UK) and normalized to the edge film thickness of each sample to compare different films easily. Additionally, we constructed organic solar cells made of poly [4,8-bis[(2-ethylhexyl)oxy]benzo[1,2-b:4,5-b'] dithiophene-2,6-diyl] [3-fluoro-2-[(2-ethylhexyl)carbonyl]thieno[3,4-b]thiophenediyl] $\left(\left(\mathrm{C}_{41} \mathrm{H}_{53} \mathrm{FO}_{4} \mathrm{~S}_{4}\right) n\right.$, PTB7): [6,6]-phenyl$\mathrm{C}_{71}$-butyric acid $\left(\mathrm{C}_{82} \mathrm{H}_{14} \mathrm{O}_{2}, \mathrm{PC}_{71} \mathrm{BM}\right)$ to investigate the effect of the thickness difference on 
the device performance. Specifically, a zinc oxide precursor solution was spin-coated on a silver-nanowire-embedded transparent electrode. After its hydrolysis process, the cosolution of PTB7:PC ${ }_{71} \mathrm{BM}$ was spin-coated on a zinc-oxide-coated transparent electrode to form a photoactive layer. Spin-coating of a diluted poly(3,4-ethylenedioxythiophene):poly(styrene sulfonate) (PEDOT:PSS) solution in isopropyl alcohol and deposition of a $100 \mathrm{~nm}$ thick silver electrode were completed to construct PTB7:PC 71 BM organic solar cells. The details were described elsewhere [28]. The device performance was evaluated by measuring the current density-voltage $(\mathrm{J}-\mathrm{V})$ characteristics under $100 \mathrm{~mW} \mathrm{~cm}^{-2}$ illumination at air mass coefficient AM 1.5 G using a solar simulator (ORIEL LCS-100, Newport) and a Keithley 2400 source meter.

\section{Results and Discussion}

To investigate the effect of polymer viscosity on the thickness difference of the silver-nanowireembedded flexible electrodes, we first used polymer SU8 to embed silver nanowires because its viscosity can be controlled while maintaining its physical properties such as the elastic modulus, strength, and hardness. Figure 2a-c shows the viscosity of SU8 as a function of the solid content and the thicknesses at the center and edge of the electrode as functions of the spin speed for different viscosities. As seen in Figure 2a, we controlled the viscosity of SU8 by tuning the solid content and successfully fabricated silver-nanowire-embedded flexible electrodes. We subsequently measured the film thickness at the center, finding higher values for increasing prepolymer solution viscosity. For example, when SU8 was coated at $500 \mathrm{rpm}$, the thicknesses were $27.5 \pm 6.2 \mu \mathrm{m}, 33.3 \pm 7.8 \mu \mathrm{m}$, $45.8 \pm 10.0 \mu \mathrm{m}$, and $75.0 \pm 23.9 \mu \mathrm{m}$ for viscosities of $52 \mathrm{cps}, 165 \mathrm{cps}, 451 \mathrm{cps}$, and 1500 cps, respectively. As the viscosity increases, the solution is increasingly difficult to spread during spin coating, and solid residuals remain on the substrate. Thus, thicker silver-nanowire-embedded transparent electrodes are obtained after delamination from the substrate. When we increased the spinning speed, the film thickness decreased, being $27.5 \pm 6.2 \mu \mathrm{m}, 16.7 \pm 7.8 \mu \mathrm{m}, 12.5 \pm 4.6 \mu \mathrm{m}$, and $11.3 \pm 3.5 \mu \mathrm{m}$ at spinning speeds of $500 \mathrm{rpm}, 1000 \mathrm{rpm}, 2000 \mathrm{rpm}$, and $3000 \mathrm{rpm}$, respectively, for a SU8 viscosity of $52 \mathrm{cps}$. This was because the centrifugal force increases at higher spinning speed, and the polymer spreads easier. The trend of the thickness at the edge was similar, but reached slightly higher values. For example, though the flexible electrode prepared with the SU8 of viscosity 52 cps had a thickness of $27.5 \pm 6.2 \mu \mathrm{m}$ at the center, its edge thickness was $71.7 \pm 11.1 \mu \mathrm{m}$. We speculate that the edge of a PMMA-coated substrate acts as a barrier, inducing prepolymer accumulation at the edge of the substrate during spin coating.

(a)

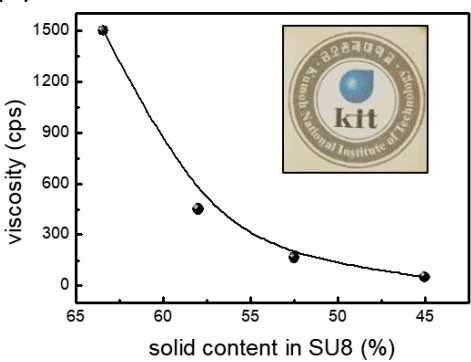

(b)

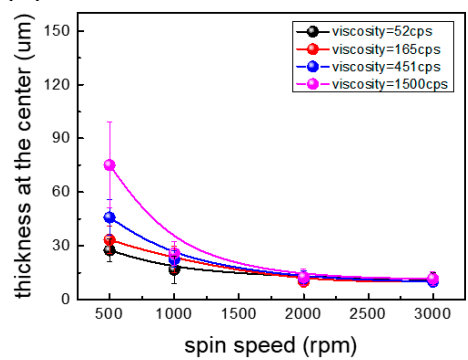

(c)

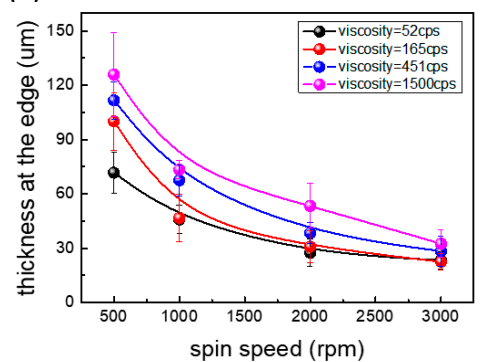

Figure 2. (a) Viscosity of the SU8 prepolymer solution as a function of the solid content and $(\mathbf{b}, \mathbf{c})$ electrode thickness at the (b) center and (c) edge as a function of the spin-coating speed for different prepolymer solution viscosities. The inset in (a) is a photograph of a SU8-embedded silver-nanowire-embedded electrode.

We then calculated the thickness difference by subtracting the thickness at the center from that at the edge. Figure 3 a shows the thickness difference as a function of the spin speed for different prepolymer solution viscosities. As can be seen, the thickness difference was high at low spin speed and low at high spin speed. For example, it was $44.2 \mu \mathrm{m}$ at $500 \mathrm{rpm}$ and $12.1 \mu \mathrm{m}$ at $3000 \mathrm{rpm}$ for a SU8 
viscosity of 52 cps. This is because a lower spin speed induces thicker films; consequently, the absolute value and difference of the thickness are high at low spin speeds. For similar reasons, the absolute value and difference of the thickness are low at high spin speeds. Therefore, to accurately compare the thickness difference among the samples with different viscosities, a normalized value is needed. Figure $3 \mathrm{~b}$ presents the thickness ratio, calculated by dividing the thickness of the center by that of the edge for all the silver-nanowire-embedded electrodes in SU8. Although the thickness ratio was slightly different for different spin speeds and viscosities, it ranged between $50 \%$ and $70 \%$, with a mean of approximately $60 \%$. This indicates the existence of a specific thickness ratio, independent of the spin speed and prepolymer solution viscosity.

(a)

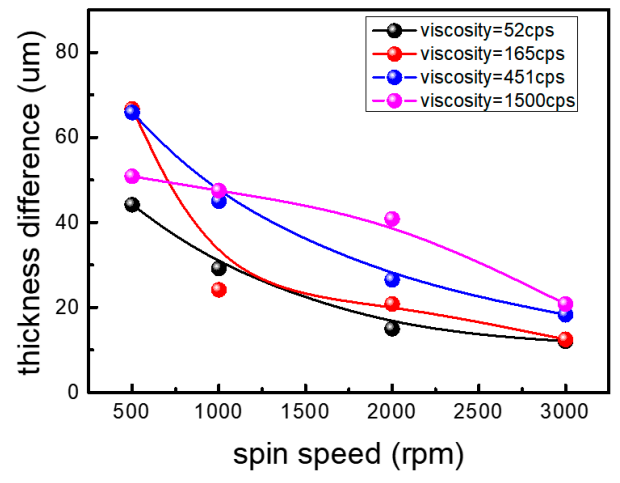

(b)

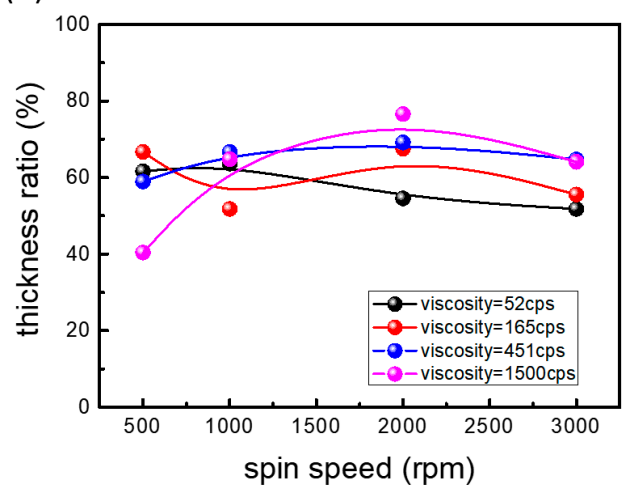

Figure 3. (a) Thickness difference and (b) thickness ratio in silver-nanowire-embedded flexible electrodes in different SU8. The thickness difference was calculated by subtracting the thickness at the edge from that at the center. The thickness ratio was obtained by dividing them by the thickness at the edge.

Next, we examined the effect of viscosity with the NOA series (NOA63, NOA65, NOA71, and NOA73) polymers, which are the most common materials used to embed silver nanowires for electrodes because, in contrast with SU8, they are very transparent. First, we inspected their appearance to verify that the silver-nanowire-embedded flexible electrodes with the NOAs were well constructed. As can be seen in the inset of Figure $4 \mathrm{a}$, the flexible electrodes looked clearer than those constructed with SU8 because the NOAs are more transparent. Additionally, no tears or defects can be seen. Thus, we confirmed the successful fabrication of silver-nanowire-embedded transparent electrodes with the NOA series polymers. After preparing the electrodes, we measured the thicknesses at their center and edge, which are shown in Figure $4 b, c$, respectively. Because the NOAs have a higher viscosity than SU8 (the highest viscosity of SU8 was $1500 \mathrm{cps}$, whereas NOA63 has a viscosity of $2500 \mathrm{cps}$ ), we expected the film to be thicker using NOAs. In fact, the maximum thickness at the center was $80 \mu \mathrm{m}$ with SU8 and $180 \mu \mathrm{m}$ with NOA63. However, the trend of the thickness for different viscosities and spinning speeds was similar. The flexible electrodes became thinner with increasing spin speed, whereas high-viscosity NOA solutions induced thicker electrodes. Additionally, the edge was thicker than the center. However, it was impossible to fabricate silver-nanowire-embedded transparent electrodes at high spinning speed using NOA73, i.e., that with the lowest viscosity, because the layers were excessively thin and could not be delaminated from the PMMA-coated substrate. 
(a)

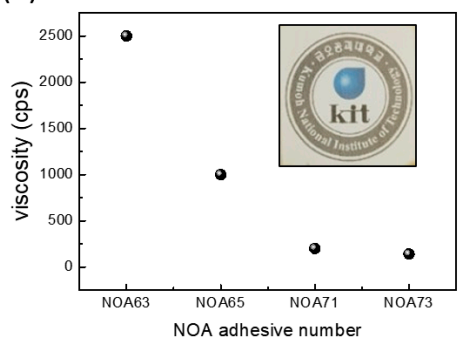

(b)

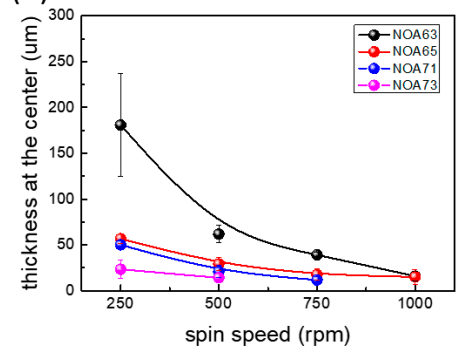

(c)

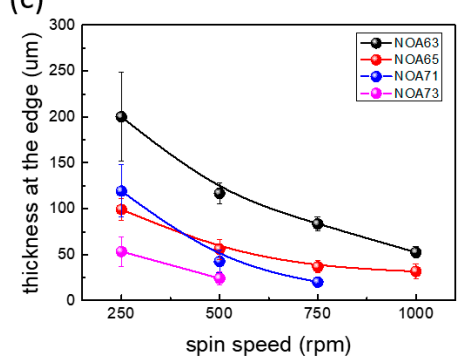

Figure 4. (a) Viscosity of the Norland optical adhesive (NOA) series solutions and (b,c) electrode thickness at the (b) center and (c) edge as a function of the spin-coating speed for different viscosities. In (a), the inset is a photograph of a silver-nanowire-embedded electrode in NOA.

We then calculated the thickness difference and ratio between the center and edge. Figure $5 \mathrm{a}, \mathrm{b}$ shows the thickness difference and ratio based on the thickness at the edge. Similar to SU8, the thickness difference decreased for increasing spinning speed in all films except for the NOA63 one. We speculate that this is because the viscosity of NOA63 was so high that the considered spinning speed range was too low. Moreover, the silver-nanowire-embedded electrodes in NOA63 showed the lowest thickness difference at a spin speed of $250 \mathrm{rpm}$. In fact, because of the high viscosity of NOA63, a low spin speed was insufficient to spread out and remove the prepolymer from the substrate. Thus, all the polymer dropped contributed to the formation of the film. A uniform dropping of NOA63 could result in a low thickness deviation. The thickness ratio of all the films appeared to be in the $40-60 \%$ range, with an average of approximately $50 \%$, except for the NOA63 one, which was spin-coated at a very low speed. Thus, we conclude that, similar to SU8, NOAs have a specific thickness ratio independent of the spinning speed and prepolymer solution viscosity, with values similar to those of SU8.

(a)

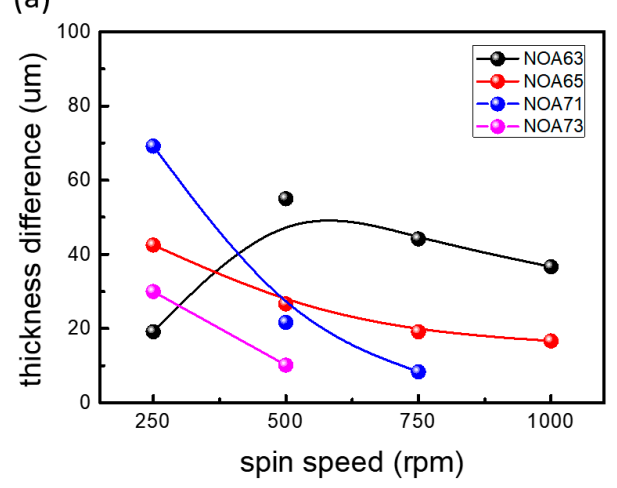

(b)

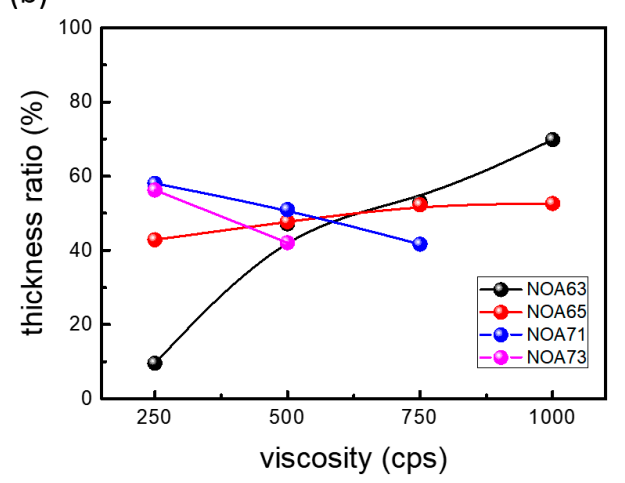

Figure 5. (a) Thickness deviation and (b) thickness ratio of silver nanowire flexible electrodes embedded in different NOAs.

Finally, we prepared PTB7:PC ${ }_{71}$ BM solar cells with silver-nanowire-embedded flexible transparent electrodes made with NOA and compared their performance at different positions with different thicknesses to examine the effect of the thickness difference on the performance. Figure 6 shows the J-V characteristics of organic solar cells located at the center and edge of silver nanowire flexible electrodes. Although their thickness was slightly different in different locations, the cells showed comparable characteristics. The open-circuit voltage and short-circuit current density of the solar cells located at the center were $0.73 \mathrm{~V}$ and $17.11 \mathrm{~mA} / \mathrm{cm}^{2}$, respectively, resulting in a power conversion efficiency of $6.12 \%$, and those of the solar cells located at the edge were $0.74 \mathrm{~V}$ and $17.82 \mathrm{~mA} / \mathrm{cm}^{2}$, resulting in a power conversion efficiency of $6.80 \%$. This indicates that the performance of the flexible electrodes is comparable and is independent of the location and thickness difference of the electrode film. When considering that a silver nanowire network is generated during spin coating on the PMMA-coated 
glass substrates, forming an electrical path, and that NOA has similar transmittance regardless of the thickness, this conclusion appears reasonable.

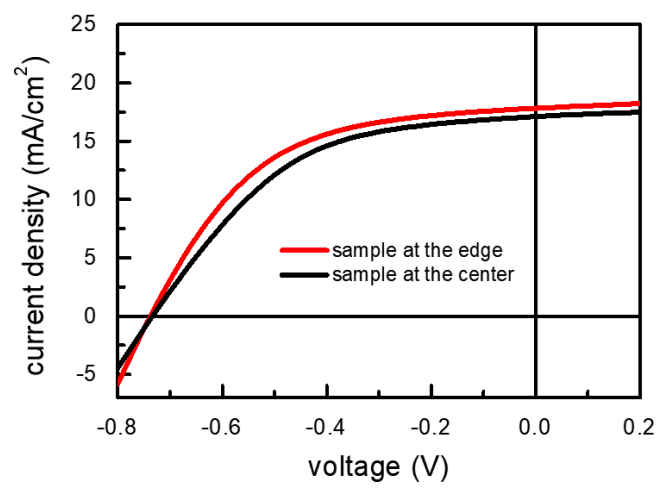

Figure 6. J-V characteristics of PTB7:PC 71 BM organic solar cells constructed at the center and edge of silver-nanowire-embedded flexible electrodes with NOA.

\section{Conclusions}

We explored the effect of the viscosity of a prepolymer solution on the thickness uniformity of silver-nanowire-embedded flexible transparent electrodes. Analysis of the SU8 polymer model system for embedding silver nanowires and of the NOA series, which are common polymers in silver-nanowire-embedded electrodes, indicated that all the electrodes had a thickness ratio of approximately $50-60 \%$. However, despite their different thicknesses, all the films showed comparable solar cell characteristics because the thickness difference did not affect the transmittance and electrical conductance of the electrodes. Thus, we conclude that single-step spin coating has limitations in constructing flexible electrodes with uniform thickness and that all the films can be applied to flexible optoelectronic devices independently of their thickness difference. In addition, multi-step spin-coating approaches, doctor blade coating, slot die coating, etc., should be considered for the formation of uniform films over a large area using high-viscosity solutions.

Author Contributions: M.C., D.K. and J.K. conceived and designed the experiments; M.C., D.K., and Y.M. conducted the experiments. S.J., D.C.H., H.-J.O. and J.K. analyzed data and contributed to the manuscript preparation. H.-J.O. and J.K. wrote the first draft of the manuscript and revised it. All authors have read and agreed to the published version of the manuscript.

Funding: This research was supported by the Kumoh National Institute of Technology, grant number 2018-104-103.

Conflicts of Interest: The authors declare no conflict of interest.

\section{References}

1. Wang, J.; Hassan, M.; Liu, J.; Yu, S. Nanowire Assemblies for Flexible Electronic Devices: Recent Advances and Perspectives. Adv. Mater. 2018, 30, 1803430. [CrossRef]

2. Gao, W.; Ota, H.; Kiriya, D.; Takei, K.; Javey, A. Flexible Electronics toward Wearable Sensing. Acc. Chem. Res. 2019, 52, 523-533. [CrossRef] [PubMed]

3. Sakamoto, K.; Kuwae, H.; Kobayashi, N.; Nobori, A.; Shoji, S.; Mizuno, J. Highly flexible transparent electrodes based on mesh patterned rigid indium tin oxide. Sci. Rep. 2018, 8, 2825. [CrossRef] [PubMed]

4. Song, J.; Son, D.; Kim, J.; Yoo, Y.J.; Lee, G.J.; Wang, L.; Choi, M.K.; Yang, J.; Lee, M.; Do, K.; et al. Wearable Force Touch Sensor Array Using a Flexible and Transparent Electrode. Adv. Funct. Mater. 2017, 27, 1605286. [CrossRef]

5. Kwon, S.R.; Elinski, M.B.; Batteas, J.D.; Lutkenhaus, J.L. Robust and Flexible Aramid Nanofiber/Graphene Layer-by-Layer Electrodes. ACS Appl. Mater. Interfaces 2017, 9, 17125-17135. [CrossRef] [PubMed]

6. Rezaie, M.N.; Manavizadeh, N.; Abadi, E.M.N.; Nadimi, E.; Boroumand, F.A. Comparison study of transparent RF-sputtered ITO/AZO and ITO/ZnO bilayers for near UV-OLED applications. Appl. Surf. Sci. 2017, 392, 549-556. [CrossRef] 
7. Lee, Y.; Kim, J.; Jang, J.N.; Yang, I.H.; Kwon, S.; Hong, M.; Kim, D.C.; Oh, K.S.; Yoo, S.J.; Lee, B.J.; et al. Development of inverted OLED with top ITO anode by plasma damage-free sputtering. Thin Solid Films 2009, 517, 4019-4022. [CrossRef]

8. Lucera, L.; Machui, F.; Kubis, P.; Schmidt, H.D.; Adams, J.; Strohm, S.; Ahmad, T.; Forberich, K.; Egelhaaf, H.-J.; Brabec, C.J. Highly efficient, large area, roll coated flexible and rigid OPV modules with geometric fill factors up to $98.5 \%$ processed with commercially available materials. Energy Environ. Sci. 2016, 9, 89-94. [CrossRef]

9. Reuillard, B.; Ly, K.H.; Hildebrandt, P.; Jeuken, L.J.C.; Butt, J.N.; Reisner, E.J. High performance reduction of $\mathrm{H} 2 \mathrm{O} 2$ with an electron transport decaheme cytochrome on a porous ITO electrode. Am. Chem. Soc. 2017, 139, 3324-3327. [CrossRef]

10. Kinner, L.; Bauch, M.; Wibowo, R.A.; Ligorio, G.; List-Kratochvil, E.J.W.; Dimopoulos, T. Polymer interlayers on flexible PET substrates enabling ultra-high performance, ITO-free dielectric/metal/dielectric transparent electrode. Mater Des. 2019, 168, 107663. [CrossRef]

11. Yang, C.-W.; Park, J.-W. The cohesive crack and buckle delamination resistance of indium tin oxide (ITO) films on polymeric substrates with ductile metal interlayers. Surf. Coat. Technol. 2010, 204, 2761-2766. [CrossRef]

12. Sierros, K.A.; Morris, N.J.; Ramji, K.; Cairns, D.R. Stress-corrosion cracking of indium tin oxide coated polyethylene terephthalate for flexible optoelectronic devices. Thin Solid Films 2009, 517, 2590-2595. [CrossRef]

13. Li, H.U.; Jackson, T.N. Flexibility Testing Strategies and Apparatus for Flexible Electronics. IEEE Trans Device Mater. Reliab. 2016, 63, 1934-1939. [CrossRef]

14. Liu, Z.; You, P.; Xie, C.; Tang, G.; Yan, F. Ultrathin and flexible perovskite solar cells with graphene transparent electrodes. Nano Energy 2016, 28, 151-157. [CrossRef]

15. Jeon, I.; Yoon, J.; Kim, U.; Lee, C.; Xiang, R.; Shawky, A.; Xi, J.; Byeon, J.; Lee, H.M.; Choi, M.; et al. High-Performance Solution-Processed Double-Walled Carbon Nanotube Transparent Electrode for Perovskite Solar Cells. Adv. Energy Mater. 2019, 9, 191204. [CrossRef]

16. Zhang, X.; Wu, J.; Wang, J.; Zhang, J.; Yang, Q.; Fu, Y.; Xie, Z. Highly conductive PEDOT:PSS transparent electrode prepared by a post-spin-rinsing method for efficient ITO-free polymer solar cells. Sol. Energy Mater. Sol. Cells 2016, 144, 143-149. [CrossRef]

17. Ko, D.; Gu, B.; Cheon, J.; Roh, J.-S.; Kim, C.S.; Jo, S.; Hyun, D.C.; Kim, J. Decoupling the contributions to the enhancement of electrical conductivity in transparent silver nanowire/zinc oxide composite electrodes. Mater. Chem. Phys. 2019, 223, 634-640. [CrossRef]

18. Lee, H.; Kim, I.; Kim, M.; Lee, H. Moving beyond flexible to stretchable conductive electrodes using metal nanowires and graphenes. Nanoscale 2016, 8, 1789-1822. [CrossRef] [PubMed]

19. Cui, Z.; Han, Y.; Huang, Q.; Dong, J.; Zhu, Y. Electrohydrodynamic printing of silver nanowires for flexible and stretchable electronics. Nanoscale 2018, 10, 6806-6811. [CrossRef] [PubMed]

20. Zhang, Y.; Guo, J.; Xu, D.; Sun, Y.; Yan, F. One-Pot Synthesis and Purification of Ultralong Silver Nanowires for Flexible Transparent Conductive Electrodes. ACS Appl. Mater. Interfaces 2017, 9, 25465-25473. [CrossRef] [PubMed]

21. Cho, S.; Kang, S.; Pandya, A.; Shanker, R.; Khan, Z.; Lee, Y.; Park, J.; Craig, S.L.; Ko, H. Large-Area Cross-Aligned Silver Nanowire Electrodes for Flexible, Transparent, and Force-Sensitive Mechanochromic Touch Screens. ACS Nano 2017, 11, 4346-4357. [CrossRef] [PubMed]

22. Sim, H.; Kim, C.; Bok, S.; Kim, M.K.; Oh, H.; Lim, G.; Cho, S.M.; Lim, B. Five-minute synthesis of silver nanowires and their roll-to-roll processing for large-area organic light emitting diodes. Nanoscale 2018, 10, 12087-12092. [CrossRef]

23. Li, Y.; Cui, P.; Wang, L.; Lee, H.; Lee, K.; Lee, H. Highly Bendable, Conductive, and Transparent Film by an Enhanced Adhesion of Silver Nanowires. ACS Appl. Mater. Interfaces 2013, 5, 9155-9160. [CrossRef] [PubMed]

24. Singh, M.; Rana, T.R.; Kim, S.; Kim, K.; Yun, J.H.; Kim, J. Silver Nanowires Binding with Sputtered ZnO to Fabricate Highly Conductive and Thermally Stable Transparent Electrode for Solar Cell Application. ACS Appl. Mater. Interfaces 2016, 8, 12764-12771. [CrossRef] [PubMed]

25. Jin, Y.; Wang, K.; Cheng, Y.; Pei, Q.; Xu, Y.; Xiao, F. Removable Large-Area Ultrasmooth Silver Nanowire Transparent Composite Electrode. ACS Appl. Mater. Interfaces 2017, 9, 4733-4741. [CrossRef] [PubMed] 
26. Nam, S.; Song, M.; Kim, D.-H.; Cho, B.; Lee, H.M.; Kwon, J.-D.; Park, S.-G.; Nam, K.-S.; Jeong, Y.; Kwon, S.-H.; et al. Ultrasmooth, extremely deformable and shape recoverable Ag nanowire embedded transparent electrode. Sci. Rep. 2014, 4, 4788. [CrossRef]

27. Joo, Y.; Byun, J.; Seong, N.; Ha, J.; Kim, H.; Kim, S.; Kim, T.; Im, H.; Kim, D.; Hong, Y. Silver nanowire-embedded PDMS with a multiscale structure for a highly sensitive and robust flexible pressure sensor. Nanoscale 2015, 7, 6208-6215. [CrossRef]

28. Ko, D.; Gu, B.; Kang, S.J.; Jo, S.; Hyun, D.C.; Kim, C.S.; Kim, J. Critical work of adhesion for economical patterning of silver nanowire-based transparent electrodes. J. Mater. Chem. A 2019, 7, 14536-14544. [CrossRef]

29. Lau, K.S.; Chin, S.X.; Tan, S.T.; Lim, F.S.; Chang, W.S.; Yap, C.C.; Jumali, M.H.H.J.; Zakaria, S.; Chook, S.W.; Chia, C.H. Silver nanowires as flexible transparent electrode: Role of PVP chain length. J. Alloys Compd. 2019, 803, 165-171.

30. Hwang, J.; Shim, Y.; Yoon, S.-M.; Lee, S.H.; Park, S.-H. Influence of polyvinylpyrrolidone (PVP) capping layer on silver nanowire networks: Theoretical and experimental studies. RSC Adv. 2016, 6, 30972-30977. [CrossRef]

31. Andrew, P.; Ilie, A. Functionalised silver nanowire structures. J. Phys. Conf. Ser. 2007, 61, 36-40. [CrossRef]

(C) 2020 by the authors. Licensee MDPI, Basel, Switzerland. This article is an open access article distributed under the terms and conditions of the Creative Commons Attribution (CC BY) license (http://creativecommons.org/licenses/by/4.0/). 